\title{
Postglaziale Klimageschichte der Schweizer Alpen
}

\section{Einleitung}

Zusammenstellungen von Daten zur postglazialen Klimageschichte der Schweizer Alpen wurden in den letzten Jahren von zOLLER (1977) und FURRER et al. (1980) sowie von Patzelt (1977) für die Österreichischen Alpen veröffentlicht. Die Kurven von zoller und PATZELT beruhen weitgehend auf pollenanalytischen Untersuchungen, ergänzt durch glazialmorphologische Befunde, die Arbeit von FURRER et al. befaßt sich dagegen weitgehend nur mit Gletscherschwankungen. $\mathrm{Da}$ in den letzten Jahren, beginnend mit den Arbeiten von RöTHLISBERGER (1976) und SCHNEEBELI (1976) über die Gletschergeschichte der Walliser Alpen, ständig neue Informationen zur postglazialen Klimageschichte gewonnen wurden, drängte sich eine Überarbeitung und Ergänzung der Postglazialchronologie auf. Insbesondere die dendroklimatologischen Untersuchungen von RENNER (1982) und BIRCHER (1982) sowie die klimageschichtliche Interpretation von Solifluktionsphasen durch FURRER (1977) und GAMPER (1981) bilden zwei neue Informationsquellen, die erst kürzlich erschlossen wurden.

Im Folgenden werden zuerst die zur Rekonstruktion des Klimas verwendeten Methoden vorgestellt und besonders im Hinblick auf deren Aussagewert bezüglich Klimaschwankungen kritisch betrachtet. Die daran anschließende Datensammlung beinhaltet die von uns berücksichtigten Befunde zur postglazialen Klimageschichte. Sämtliche Angaben, vor allem die dazugehörigen ${ }^{14} \mathrm{C}$-Daten, wurden kritisch durchgesehen. Alle nicht eindeutig interpretierbaren Angaben, zum Beispiel Pollenschwankungen, die nur bedingt klimatisch verursacht sein könnten, oder ${ }^{14} \mathrm{C}$-Alter von Hölzern aus Gletschervorfeldern, die nicht unbedingt mit Gletscherschwankungen im Zusammenhang stehen, wurden eliminiert. Grundsätzlich wurden auch alle ${ }^{14} \mathrm{C}$-Alter mit Standardabweichungen von über 120 Jahren nicht verwendet. Die aus dieser Datensammlung resultierende Kurve der Gletscherschwankungen (Fig. 1) basiert dennoch auf 103, die Gliederung der Solifluktionsphasen auf 85 und die dendroklimatologische Gliederung auf $47{ }^{14} \mathrm{C}$-Daten. Der jüngste Teil des Postglazial nach $700 \mathrm{BP}$ wurde in der vorliegenden Arbeit nicht mehr berücksichtigt, da in diesem Zeitraum vorwiegend mit Kalenderdaten gearbeitet werden kann. Ein direkter Vergleich mit den hier zusammengestellten Daten, basierend auf Radiocarbonaltern, ist deshalb nicht möglich.

\section{Die Interpretation von Befunden zur Klimageschichte}

\subsection{Gletscherschwankungen}

Die im Postglazial datierten Gletscherschwankungen ergeben im Prinzip eine Kurve der Zungenlängenänderungen. Bei deren Interpretation gilt es nun aber, einige Punkte zu beachten: Auf eine Änderung der beteiligten Klimafaktoren paßt sich am Gletscher nur die Gleichgewichtslinie direkt und ohne zeitliche Verzögerung an. Dabei gilt für zentralalpine Verhältnisse, daß eine Absenkung der Gleichgewichtslinie um $100 \mathrm{~m}$ entweder verursacht wird durch eine erhöhte Winterakkumulation um $400 \mathrm{~kg} \mathrm{~m}^{-2}$, durch die Absenkung der Sommertemperatur um $0,8^{\circ} \mathrm{C}$ oder durch die Reduktion der sommerlichen Strahlungsbilanz um $1,3 \mathrm{MJ} \mathrm{m}^{-2} \mathrm{~d}^{-1}$ (KUHN 1979, 18). Es ist jedoch zu bedenken, daß die drei Größen selbstverständlich nicht völlig unabhängig voneinander sind Zudem ist bei der Akkumulation neben dem festen Niederschlag der Anteil der Schneeverfrachtung, welcher jährlich starken Schwankungen unterworfen ist, nicht zu unterschätzen. So macht beispielsweise am Hintereisferner der Driftschnee rund 50\% der Akkumulation aus (KUHN 1981).

Wie und nach welcher Zeit wirkt sich nun aber eine Massenänderung, die in direkter Beziehung zur Höhenänderung der Gleichgewichtslinie steht (HOINKES 1970, 68), an der Gletscherzunge aus? Dies hängt, abgesehen von den Klimaparametern, einerseits von den Materialeigenschaften des Eises, anderseits von der Gletschergeometrie, die teilweise durch die Topographie bestimmt ist, ab (PATERSON 1969, 195ff.); Größen, die selbstverständlich für jeden Gletscher verschieden sind. Daneben sind auch autokorrelative Prozesse nicht zu vernachlässigen (PATERSON 1969, 230; suter unpubl.). Allgemein kann aber gesagt werden, daß kleine Gletscher auf Massenänderungen an der Zunge rascher, größere langsamer, ganz große oft mit einer derartigen Verspätung reagieren, daß die Klimatendenz zuweilen bereits wieder umgeschlagen hat. Damit spiegeln kleine Gletscher kurzfristige

Dr. Martin Gamper und Dr. Jürg Suter

Geographisches Institut der Universität Zürich,

Postfach, 8033 Zürich 
Veränderungen wieder, große Eisströme dagegen sprechen nur auf langfristige Änderungen an (HANTKE 1978, 53).

Weiter gilt es zu beachten, daß die Sommertemperaturen der einzelnen Klimastationen über den Alpenraum wohl sehr hoch korrelieren, der Winterniederschlag aber nur innerhalb bestimmter Regionen (z. B. Südstaubereich) eine hohe Korrelation aufweist (suTER 1982). Dadurch wird die Vergleichbarkeit der einzelnen Gletscherdaten weiter erschwert.

Diesen Umständen (regionale Gliederung, gletscherspezifische Größen) wird insofern Rechnung getragen, indem bei der Behandlung der einzelnen Zeiträume die Gletschernamen sowie die zugehörigen Regionen aufgeführt sind, wodurch eine Eigenbeurteilung des klimatischen Ereignisses ermöglicht wird.

Daß nun in Fig. 1 trotzdem eine Kurve der Gletscherschwankungen gezeichnet wurde, läßt sich aufgrund der Untersuchungen über die Neuzeit rechtfertigen (FURRER et al. 1980, 97): Es zeigte sich, daß alle Gletscher immer etwa das gleiche "Niveau» aufweisen, d.h. daß trotz den individuellen Abweichungen nicht ein Teil der Gletscher eine Ausdehnung wie um 1973 zeigt, während gleichzeitig der Rest einen Hochstand aufweist. Als Bezugspunkte für die Kurve dienten die Gletscherstände um 1850, 1890, 1920 sowie die Ausdehnung von 1973 (Gletscherinventar: MÜLLER et al., 1976), wie sie schon von SCHNEEBELI und RÖTHLISBERGER $(1976,134)$ verwendet wurden.

\subsection{Fossile Böden in Seiten- und Endmoränen}

Gletschervorstöße können mit fossilen Böden unter Moränen datiert und rekonstruiert werden. Im Bereich der Schweizer Alpen wurden solche fossile Böden erstmals in den Arbeiten von RÖTHLISBERGER (1976), SCHNEEBELI (1976) sowie MüLLER (1975) datiert und ausgewertet.

Später wurden auch in anderen Gebieten der Schweizer Alpen weitere fossile Böden unter Moränenmaterial gefunden und datiert. Eine Zusammenstellung dieser vorwiegend in nicht veröffentlichten Diplomarbeiten aus dem Geographischen Institut der Universität Zürich enthaltenen Daten findet sich in RöTHLISBERGER et al. (1980).

Bei allen diesen Arbeiten ergaben sich Probleme bei der Interpretation der ${ }^{14} \mathrm{C}$-Alter dieser Böden. RöTHLISBERGER et al. (1980, S. 25) schreiben dazu, daß Anfang und Ende der Bodenbildungsphasen unbekannt bleibe; das scheinbare Bodenalter liege jedoch um so näher beim Alter der Überschüttung durch den Gletscher, je kürzer die Bodenbildungsphase gewesen war.

GAMPER (1982a) datierte deshalb aus der von BLESS (1982) glazialmorphologisch und klimageschichtlich bearbeiteten Seitenmoräne des Glacier d'Argentière die Huminsäuren, die organische Restsubstanz (= Humine und makroskopisch nicht sichtbare Pflanzen- reste) sowie Holz aus sechs entsprechenden übereinanderliegenden fossilen Böden.

Die statistische Auswertung dieser Daten ergab keine signifikanten Altersunterschiede zwischen den drei verschiedenen Kohlenstofffraktionen (GAMPER und OBERHÄNSLI 1982). Dies bedeutet einerseits, daß die fossilen Böden nicht durch jüngere Huminsäuren und Wurzeln kontaminiert wurden, da die Alter der beiden Huminstofffraktionen dem Alter der im Boden eingeschlossenen Holzstücke entsprechen; andererseits zeigen diese Ergebnisse, daß die Bodenbildungsphasen alle sehr kurz gewesen sein müssen, da keine Altersunterschiede zwischen den Huminsäuren und den organischen Restsubstanzen bestehen. In den meisten Fällen würde aber trotzdem erst eine getrennte Datierung von Huminsäuren und organischer Restsubstanz eine sinnvolle und genügend abgesicherte Beurteilung des Bodenalters ermöglichen, da erst der Vergleich dieser beiden Alter mögliche Verunreinigungen durch junge Huminsäuren oder alten, fremden Kohlenstoff erkennen läßt (GAMPER Und OBERHÄNSLI 1982).

\subsection{Holzfunde über der heutigen potentiellen Waldgrenze}

Durch den Einfluß der Alpwirtschaft und früher auch als indirekte Folge des Bergbaus liegt die aktuelle Waldgrenze meist tiefer als die potentielle Waldgrenze. Für die Wertung der Höhenlage eines Holzfundes bezüglich der Waldgrenze gilt es daher, in jedem Fall zuerst die potentielle Waldgrenze im Untersuchungsgebiet festzulegen. Dazu hat sich die obere Grenze des geschlossenen Alpenrosengürtels als guter Näherungswert erwiesen (HAGER 1916, 85; LANDOLT 1969, 46). Für die Lage der Waldgrenze sind, vom klimatischen Gesichtspunkt her, die sommerlichen Temperatursummen entscheidend. So genügen 3 bis 10 Jahre unterdurchschnittliche Werte, um die Waldgrenze abzusenken (BORTENSCHLAGER 1977, 263). Für einen Anstieg darf sicher eine Erhöhung der sommerlichen Temperatursummen angenommen werden. Jedoch ist die dafür erforderliche Zeitspanne noch kaum bekannt. Zudem darf der Einfluß von nichtklimatischen Faktoren, die sich negativ auf einen Waldgrenzanstieg auswirken, nicht unterschätzt werden.

Beim Vergleich der Lage «Holzfund zur potentiellen Waldgrenze» gilt allgemein: Da Pflanzengesellschaften auf Klimaverbesserungen sehr langsam reagieren, ergibt sich durch die Bestimmung der potentiellen Waldgrenze mit Hilfe der geschlossenen Alpenrosenbestände eine Höhenlage, welche die klimatischen Verhältnisse der letzten Jahrzehnte ausdrückt. Dadurch muß angenommen werden, daß nur Holzfunde von mehr als $100 \mathrm{~m}$ über der potentiellen Waldgrenze auf ein wärmeres Klima als heute deuten (TURNER in SU'TER 1981, 34). Diese Befunde sind in der Kurve der Gletscherschwankungen (Fig. 1) mitberücksichtigt. 


\subsection{Schwankungen der solifluidalen Aktivität von Erdströmen}

Mit Hilfe der ${ }^{14} \mathrm{C}$-Datierung von fossilen Böden aus Erdströmen können klimageschichtliche Informationen gewonnen werden (vgl. FURRER et al. 1975, FURRER 1977, BEELER 1977, GAMPER 1981). Bei Erdströmen handelt es sich um zungenförmige Ausläufer von Solifluktionsdecken.

Diese Solifluktionsform findet sich in den Alpen auf einer Höhe von 2300 bis 2500 Metern, also in der subnivalen Höhenstufe am Übergang vom alpinen Rasen zum Frostschutt. Die klimatischen Verhältnisse und damit die klimageschichtlichen Informationen, die bei der Untersuchung von Erdströmen anfallen, sind deshalb mit den Resultaten gletschergeschichtlicher Arbeiten vergleichbar.

Die heutigen Solifluktionsbeträge und der Bewegungsmechanismus der Erdströme müssen bekannt sein, um beurteilen zu können, ob überhaupt eine $\mathrm{Zu}$ und Abnahme der solifluidalen Tätigkeit notwendig ist, damit eine Wechsellagerung von fossilen Böden und Solifluktionsschutt entsteht; d.h. die klimatische Steuerung muß nachgewiesen werden.

Die heutigen solifluidalen Bewegungen von Erdströmen wurden von FURRER $(1954,1972)$, FURRER et al. (1971) und GAMPER $(1976,1981)$ im Schweizerischen Nationalpark untersucht. Die von GAMPER in den Jahren 1975-1979 durchgeführten Bewegungs- und Temperaturmessungen an Erdströmen ergaben, da $\beta$ sich die Erdströme im Nationalpark heute in einer Ruhephase befinden. Insbesondere vollständig bewachsene Erdströme sind inaktiv, die übrigen Zungen zeigen nur Bewegungen auf ihren vegetationsfreien Rücken, die Steilränder sind stabil (GAMPER 1981, S. 405/406).

Eine wesentliche Verstärkung der Solifluktion ist zu erwarten, wenn kalte und nasse Sommer sowie kalte Winter häufiger auftreten (GAMPER 1981, 406). Im Engadin und im Gebiet des Nationalparks, wo diese Untersuchungen durchgeführt wurden, würde diese Klimaänderung bei einer übernormalen Häufigkeit von Nordlagen eintreten (vgl. GENSLER 1978, S.96). Fossile, datierte Hinweise auf eine verstärkte Solifluktion während des Postglazials in den Alpen deuten deshalb darauf hin, $d a ß$ in solchen Zeiträumen häufiger als normal Nordwest- und Nordlagen auftraten, d.h. allgemein also eine Klimaverschlechterung eintrat (GAMPER 1981).

Die Datierung von fossilen Bodenbildungen unter Solifluktionszungen, der zweite, wichtige Problemkreis im Zusammenhang mit deren klimageschichtlicher Auswertung, wurde im Bereich der Schweizer Alpen von FURRER (1954) aufgegriffen und später ausführlicher behandelt (FURRER et al. 1971 und 1975, FURRER 1972 und 1977).

In den folgenden Jahren wurden diese Untersuchungen durch GAMPER im Berninapaßgebiet und im Nationalpark fortgesetzt. Im Rahmen dieser Arbeit wurden ${ }^{14} \mathrm{C}$-Alter von 35 Bodenproben bestimmt (GAMPER 1981).

Aus diesen Arbeiten ergab sich eine Gliederung der letzten 5000 Radiocarbonjahre (Fig. 1) in Zeiten verstärkter solifluidaler Aktivität und Zeiten morphologischer Ruhe von Erdströmen (GAMPER 1981, S. 429).

Zusammenfassend kann festgestellt werden, daß die Prozesse und deren klimatische Steuerung, die zu einer Verstärkung der Solifluktion führen, durch die Untersuchungen im Schweizerischen Nationalpark weitgehend bekannt sind (GAMPER 1981). Die Datierung und Interpretation fossiler Böden aus Erdströmen können dabei durch fraktionsweise Datierung von Huminstoffen zusätzlich abgesichert werden (GAMPER und OBERHÄNSLI 1982). Weitere Informationen über die heutigen solifluidalen Prozesse in den Alpen sowie zusätzliche Datierungen fossiler Böden in anderen Gebieten der Alpen sind aber notwendig und können unsere Kenntnisse über die solifluidale Aktivität und damit über das Klimageschehen während des Postglazials noch wesentlich erweitern und verfeinern.

\subsection{Dendroklimatologie (nach RENNER, 1982)}

Die in Fig. 1 dargestellte dendroklimatologische Gliederung entstammt 11 einzelnen, ${ }^{14} \mathrm{C}$-datierten, sogenannten schwimmenden Chronologien, wie sie von BIRCHER (1982) und RENNER (1982) auf der Basis der Radiodendroklimatologie (maximale Dichten) erarbeitet wurden. Die einzelnen Mittelkurven der Chronologien sind über weite Strecken aus mindestens 3 verschiedenen Baumkurven zusammengesetzt. Einzelne Zeitabschnitte sind 5- bis 6fach belegt, andere, vor allem Beginn und Ende der Mittelkurven, nur 1bis 2 fach. Jede «Baumkurve» entspricht dabei einer Mittelkurve von mindestens 2 Radien. $\mathrm{Zu}$ beachten ist, da $\beta$ die Mittelkurven von unterschiedlichen Wachstumsstandorten und aus verschiedenen Zeitperioden stammen. Der Mittelwert jeder einzelnen Chronologie entspricht deshalb den durchschnittlichen Wachstumsbedingungen, die während der entsprechenden Zeitperiode geherrscht haben. Streng genommen gelten somit klimatische Aussagen, die aus schwimmenden Chronologien gewonnen werden, immer nur für den Zeitraum, der durch die Chronologie abgedeckt ist. Aufgrund bisheriger Erkenntnisse über das Postglazial kann aber angenommen werden, daß die Mittelwerte der mehrhundertjährigen Chronologien nahezu gleich sind. Bei kürzeren Reihen sind allerdings größere Abweichungen nicht auszuschlieBen. Trotz der bedingten Vergleichbarkeit (unterschiedliche Belegungsdichte, Mittelwerte, Amplituden) der einzelnen Chronologien können, aufgrund der bisherigen Untersuchungen an rezenten Hölzern aus ebenfalls subalpinen kaltfeuchten Lagen, langdauernde Perioden unterdurchschnittlicher maximaler - Dichten als kühle bis kalte Zeitabschnitte bezeichnet 
werden; Perioden überdurchschnittlicher Dichte dagegen als warme Zeitabschnitte. Dabei beziehen sich die Aussagen «kalt», "kühl» und "warm» auf die Sommermonate Juli bis September (vgl. SCHWEINGRUBER 1976; SCHWEINGRUBER et al. 1978, 1979; BIRCHER 1982). Für weitere Hinweise zur Kurveninterpretation (z. B. Sensitivität) sei auf die Arbeit von F.RENNER (1982) verwiesen.

In Figur 1 gelangen nur diejenigen Zeitabschnitte zur Darstellung, in denen die gefilterten Mittelkurven während mindestens 50 Jahren unter- oder oberhalb des Mittelwertes verlaufen. Die Filterung basiert dabei auf einem Low Pass-Filter (Binomialfilter, 31 gewichtete Glieder).

\subsection{Pollenanalyse}

Postglaziale Klimaschwankungen zeichnen sich besonders in der subalpinen Höhenstufe auch im Wechsel der Vegetation ab. Pollenanalytische Untersuchungen von Mooren in dieser Höhenlage können deshalb auch zur Rekonstruktion der Klimageschichte verwendet werden. Änderungen im Vegetationsbild und Klimaschwankungen müssen jedoch nicht immer miteinander in Korrelation stehen. c. BURGA (1979, S. 266-267) schreibt dazu: «Dabei sind vor allem zu beachten das Konkurrenzverhalten und die vom Relief und dessen Substrat vorgegebenen Einwanderungsmöglichkeiten der Pflanzen während des Spät- und Postglazials und die Trägheit der Ökosysteme (vgl. auch LANG 1970 und zOLLER 1968). Zudem können gleiche Erscheinungen verschiedene Ursachen im biotischen und abiotischen Bereich aufweisen. Manche Klimaschwankungen kommen nur in bestimmten Höhenzonen (zoLLER 1960) oder nur in gewissen Landschaften zum Ausdruck (FRENZEL 1975, LANG 1952). Eine vegetative Pflanzenvermehrungsphase kann als Klimaschwankung gedeutet werden (FRENZEL 1977). Deshalb warnten FAEGRI und IVERSEN (1975) vor Schlüssen, die gezogen werden in Pollendiagrammen über das Fehlen von bestimmten Pollentypen.»

Außerdem ist in der zweiten Hälfte des Postglazials mit zunehmendem anthropogenem Einfluß zu rechnen.

\section{Zusammenstellung bisheriger Ergebnisse}

\section{$10200-9500 B P$}

Für diesen Zeitabschnitt liefert im wesentlichen nur die Pollenanalyse einige Hinweise, wobei aber die Meinungen über deren klimageschichtliche Auswertung stark auseinandergehen (ZOLLER 1960; KÜTTEL 1977, 79; BEHRE 1978). So können die präborealen Oszillationen als der Klimadepression der Jüngeren Dryas nachhinkende Reaktion der Vegetation aufgefaßt werden, wobei auch eine verzögerte Vegetationsentwicklung mit zunehmender Höhenlage in Rech- nung zu stellen ist. Daneben könnte aber unmittelbar nach der Jüngeren Dryas nochmals eine kurze, markante Klimadepression eingetreten sein, so daß die Vegetation sich noch nicht erholen konnte (BURGA 1979, 273).

In diesen Zeitraum ist auch die Palü-Schwankung zu setzen, welche sich kurz vor 9460 BP abgespielt hat. Der Palügletscher dürfte dabei in der Schlucht zwischen Alpe Palü und Cavaglia gestirnt haben (BEELER 1977, 213); d.h. die 1850-Ausdehnung wurde noch deutlich überschritten.

\section{0-8500 BP}

Spätestens zu Beginn dieses Zeitraumes schmolzen die Alpengletscher auf neuzeitliche Größenordnung ab: Palügletscher um 9460 BP (BEELER 1981, 104).

Mit der Datierung der äußersten Jahrringe eines 276jährigen Lärchenstammes aus dem Vorfeld des Glacier de Ferpècle liegt für den Alpenraum das bisher älteste Datum $(9010 \pm 100 \mathrm{BP})$ vor, daß ein Gletscher erstmals im Postglazial kleiner als um 1850 A.D. (in diesem Fall sogar kleiner als um 1920 A.D.) war (RÖTHLISBERGER et al. 1980, 42).

\section{$8500-7500 B P$}

Dieser Zeitraum ist gekennzeichnet durch Gletschervorstöße im Wallis nach $8400 \mathrm{BP}$, Klimarückschläge in Pollenprofilen im Gotthardgebiet und Graubünden und anschließende Erwärmung mit Gletscherausdehnung kleiner als 1920 A.D. (Wallis).

Gletscher: Nach 8400 BP Vorstöße des Tsidjiore Nouve und Gornergletscher im Wallis (RÖTHLISBERGER 1976, 137). Von 7700 BP bis 7400 BP ein oder zwei Gletschervorstöße im Wallis nach einer Zeit allgemein geringer Ausdehnung (RÖTHLISBERGER 1976, 137; RÖTHLISBERGER et al. 1980, 42). Diese Vorstöße stehen möglicherweise bereits im Zusammenhang mit den Misoxerkaltphasen.

Waldgrenze: Um 8085 BP mindestens so hoch wie heute (Engadin, sUTER 1981, 55).

Dendroklimatologie: Warmphasen von 7740 BP bis $7685 \mathrm{BP}$ und $7610 \mathrm{BP}$ bis $7560 \mathrm{BP}$; Kaltphasen von $7830 \mathrm{BP}$ bis $7740 \mathrm{BP}$ und $7685 \mathrm{BP}$ bis $7610 \mathrm{BP}$ (RENNER 1982, 157).

Pollen: Oberhalbsteinschwankung um 7750 BP im Sinne einer merklichen Abkühlung und erhöhter Niederschläge (HEITZ 1975, 22). In mehreren Profilen nachgewiesen (HEITZ 1975, 55).

\section{$7500-6000 \mathrm{BP}$}

Diese Phase ist geprägt durch einen länger anhaltenden Klimarückschlag von $7500 \mathrm{BP}$ bis etwa $6400 \mathrm{BP}$, der im wesentlichen nur pollenanalytisch nachgewiesen werden kann.

Gletscher: Im Wallis Vorstöße um 7400 BP (siehe Abschnitt $8500 \mathrm{BP}$ bis $7500 \mathrm{BP}$ ). 
Solifluktion: Nach 6850 BP Anzeichen für Solifluktion im Nationalpark (Engadin, FURRER 1977, 269).

Dendroklimatologie: Warmphase von $7140 \mathrm{BP}$ bis 7080 BP, Kaltphasen von 7250 BP bis 7190 BP, 7020 BP bis $6960 \mathrm{BP}$ und $6550 \mathrm{BP}$ bis $6480 \mathrm{BP}$ (RENNER 1982, 158).

Pollen: Misoxerkaltphasen zwischen 7500 BP und 6500 BP: mehrmalige Waldauflockerung; Kältrückfälle, die vorübergehend die obere Grenze des Baumwuchses und den Abies-Gürtel um höchstens 200-250 m herabgedrückt haben (zoller 1960, 82). Diese Schwankung ist auch in verschiedenen anderen Profilen nachgewiesen worden (vgl. BURGA 1979, 270/271).

\section{$6000-4400 B P$}

Im Gegensatz zu den Befunden aus den Österreichischen Alpen (Patzelt 1973, 63) fanden sich in den Schweizer Alpen zwischen 6400 BP und 5750 BP bisher keine Hinweise auf Klimaverschlechterungen. Nach einer ersten Phase geringer Holzdichte von 5745 BP bis 5695 BP setzt um 5400 BP die Piorakaltphase ein (belegt durch Pollenanalysen, Gletschervorstöße, Solifluktionsphasen und Zeiträume mit geringer Holzdichte). Zwischen $5000 \mathrm{BP}$ und $4500 \mathrm{BP}$ wurde diese Kaltphase mindestens einmal unterbrochen, mit anschließender Klimaverschlechterung bis 4400 BP.

Gletscher: Zwischen 5300 BP und $4400 \mathrm{BP}$ sind an verschiedenen Gletschern der gesamten Schweizer Alpen Vorstöße belegt: Maighels (Gotthardgebiet, RENNER 1982, 58) Gornergletscher (Wallis, RÖTHLISBERGER 1976, 88), Oberer Grindelwaldgletscher (Berner Oberland, RöTHLISBERGER 1976, 95), Oberaar (Berner Oberland, RÖTHLISBERGER et al. 1980, 50) und Calderas (Engadin, suTER 1981, 50). Einzig im Engadin konnten am Tschiervagletscher zwei zeitlich sicher getrennte Vorstöße nachgewiesen werden (um 5160 BP und 4510 BP, GAMPER-SCH. 1982), wobei der Gletscher beidemal zuvor kleiner als heute war. Häufig wurde bei den Vorstößen in diesem Zeitraum die Ausdehung von 1850 A.D. überschritten.

Solifluktion: Im Engadin verstärkte Solifluktion zwischen 4600 BP und 4300 BP (FURRER 1977, 269; BEELER 1977, 137; GAMPER 1981, 426). Vorher und nachher sind Bodenbildungsphasen belegt. Auch im Wallis nach 4500 BP Hinweis auf Solifluktion (RÖTHLISBERGER 1976, 138).

Dendroklimatologie: Warmphasen von 5605 BP bis $5525 \mathrm{BP}$ und $5065 \mathrm{BP}$ bis $4960 \mathrm{BP}$, Kaltphasen von $5745 \mathrm{BP}$ bis $5695 \mathrm{BP}, 5275 \mathrm{BP}$ bis $5150 \mathrm{BP}$ und $4960 \mathrm{BP}$ bis 4855 BP. Während der Piora Kaltphase II ist die Kurve unterbrochen (RENNER 1982, 159).

Pollen: Zweigeteilte Piora Kaltphase (zOLler 1960, 85; ZOLLER et al. 1966, 158ff.) zwischen 5400 BP und 4000 BP. Das Ende der Schwankung wurde von HEITZ $(1975,56)$ im Profil Julier mit 4510 BP neu datiert, was gut mit den Ergebnissen des folgenden Abschnittes übereinstimmt. Die Piora Kaltphase wurde auch in mehreren andern Profilen der Schweizer Alpen nachgewiesen (BURGA 1979, 270).

\section{0-3600 BP}

Dieser Zeitraum stellt nach den bisherigen Ergebnissen das Klimaoptimum des Postglazials in den Schweizer Alpen dar.

Gletscher: Keine Anzeichen für Gletschervorstöße. Ferpècle (Wallis) um $4000 \mathrm{BP}$ kleiner als 1973 A.D. (RÖTHLISBERGER 1976, 90).

Waldgrenze: Mindestens $100 \mathrm{~m}$ höher als heute um 4355 BP (Mittelbünden, MAISCH 1981, 88), 4200 BP (Wallis, RöThlisBerger 1976, 73) und 4010 BP (Engadin, GAMPER 1981, 426). Mindestens so hoch wie heute um 4260 BP (Engadin, sUter 1981, 55) 3935 BP (Mittelbünden, FURRER et al. 1980, 101) und 3515 BP (Mittelbünden, FURRER et al. 1980, 101).

Solifluktion: Nach 4300 BP bis 3450 BP keine Anzeichen für Solifluktion (Engadin, G AM PER 1981, 426).

Dendroklimatologie: In diesen Zeitraum fällt auch die längste überdurchschnittliche Wärmeperiode im ganzen Alpenraum von 3740 BP bis 3600 BP. Weitere Warmphasen: $4395 \mathrm{BP}$ bis $4330 \mathrm{BP}, 3595 \mathrm{BP}$ bis 3535 BP. Kühlere Abschnitte: 4130 BP bis $4070 \mathrm{BP}$, $3830 \mathrm{BP}$ bis $3740 \mathrm{BP}$ (RENNER 1982, 160).

Pollen: Keine eindeutigen Hinweise auf Schwankungen.

\section{$3600-2900 B P$}

Nach der ausgeprägten Warmphase um 4000 BP erfolgte um $3300 \mathrm{BP}$ der wohl kälteste Abschnitt des Postglazials. Zwischen $3340 \mathrm{BP}$ und $3175 \mathrm{BP}$ ist dendroklimatologisch die längste und extremste Kaltphase festzustellen, die in den bisher vorhandenen Kurven beobachtet werden konnte (RENNER 1982, 162). Gleichzeitig wurde die Solifluktion verstärkt, und die Gletscher stießen vor. Um 2800 BP scheint kurzfristig eine Klimabesserung stattgefunden zu haben (Unterbruch der Solifluktion und Waldgrenzanstieg in Graubünden).

Gletscher: In der rechten Seitenmoräne des Glacier d'Argentière (Mt. Blanc) konnten drei übereinanderliegende fossile Böden datiert werden. Sie belegten Gletschervorstöße nach 3600 BP und zweimal kurz nacheinander nach 3400 BP (BLESS 1982). In die gleichen Zeiträume fallen auch Vorstöße im Wallis des Glacier de Ferpècle (RÖTHLISBERGER et al. 1980, 43), des Tsidjiore Nouve (RÖTHLISBERger et al. 1980, 44) und des Allalin (BIRCHER 1982). Auch im Gotthardgebiet konnten am Wittenwasserngletscher 2 Vorstöße nach $3600 \mathrm{BP}$ und $3500 \mathrm{BP}$ sowie am Guspisfirn nach 3565 BP und 3250 BP (RENNER 1982, 53) nachgewiesen werden. Der Steingletscher stieß auch nach 3490 BP (KING 1974, 48) und zwischen $3040 \mathrm{BP}$ und $2820 \mathrm{BP}$ vor (KING 1974, 76), wobei er in beiden Fällen die Marke von 1850 A.D. überschritt. Gletschervorstöße um $3000 \mathrm{BP}$ sind auch am Stellibodengletscher im 
Gotthardgebiet (RENNER 1982), Allalin (BIRCHER 1982) und Tsidjiore Nouve (Röthlisberger 1976, 91) im Wallis belegt.

Waldgrenze: In Mittelbünden Waldgrenze um 2920 mindestens so hoch wie heute (MAISCH 1981, 88).

Solifluktion: Im Berninagebiet und am Albulapaß (GAMPER 1982b) muss der ganze Zeitraum von $3450 \mathrm{BP}$ bis $2200 \mathrm{BP}$ als Kaltphase mit ausgeprägter Solifluktion betrachtet werden. Einzig um 2865 BP bis $2750 \mathrm{BP}$ und von $2350 \mathrm{BP}$ bis $2300 \mathrm{BP}$ sind kurze Unterbrüche festzustellen (GAMPER 1981, 426).

Dendroklimatologie: Warmphasen von 3415 BP bis $3340 \mathrm{BP}$ und $3175 \mathrm{BP}$ bis $3055 \mathrm{BP}$, Kaltphase von 3340 BP bis 3175 BP (RENNER 1982, 161).

Pollen: Die Löbbenschwankung (PATZELT und BORTENSChlager 1973, 62) ist in den Pollenprofilen der Schweiz wohl angedeutet, aber nicht sicher belegt; anthropogener Einfluß seit dem Subboreal zunehmend stärker (BURGA 1979, 269).

\section{0-2200 BP}

Nach einer kurzen Klimabesserung sind in der zweiten Hälfte dieses Abschnittes wiederum vermehrt Hinweise für Klimarückschläge vorhanden.

Gletscher: Einzig am Trient (Wallis) wurde eine Überschüttung mit einem Alter von 2770 BP (sCHNEEBELI 1976, 51) gefunden. Die nächste ausgeprägte Vorstoßphase fällt in den Zeitraum von $2550 \mathrm{BP}$ bis 2300 BP: Wallis: Findelen (RÖTHLISBERGER 1976, 63-71), Ferpècle (RÖTHLISBERGER 1976, 79/90; RÖTHLISBERGER et al. 1980, 45); Gotthardgebiet: Guspisfirn (RENNER 1982, 54); Mt. Blanc: Argentière (BLESS 1982); Engadin: Traunter Ovas (suTER 1981, 52).

Solifluktion: Im Zeitraum von $2350 \mathrm{BP}$ bis $2300 \mathrm{BP}$ finden sich im Engadin (FURRER 1977, 269; FURRER et al. 1975, 25; GAMPER 1981, 419) und im Wallis (RÖTHLISBERGER 1976, 139) Anzeichen für einen Unterbruch der solifluidalen Aktivität (vgl. Abschnitt $3600 \mathrm{BP}$ bis 2900 BP).

Dendroklimatologie: Warmphasen von 2640 BP bis 2570 BP und 2490 BP bis 2430 BP. Kaltphasen von $2850 \mathrm{BP}$ bis $2795 \mathrm{BP}, 2700 \mathrm{BP}$ bis $2640 \mathrm{BP}$ und $2570 \mathrm{BP}$ bis 2490 BP. Von 2400 BP bis 2100 BP sind ausgeglichene Verhältnisse, mit jedoch unbekanntem «Temperaturniveau» nachgewiesen (RENNER 1982, 161-164).

Pollen: Göschener Kaltphase I 2880 BP-2320 BP: Es fehlt der sichere stratigraphisch-geomorphologische Nachweis, daß die Vorstöße des Dammagletschers und der Hängegletscher nördlich des Feldschijen dieser Kaltphase zuzuordnen sind (zOLLER et al. 1966, 160). Insbesondere ist aber der oft zitierte Vorstoß des Chelengletschers 3-4 km über den neuzeitlichen Hochstand hinaus auf nicht klimatisch bedingte Ereignisse (Bergsturz mit nachfolgendem Gletscherrutsch) zurückzuführen (zoLler et al. 1966, 114-119) und ist zeitlich nach der Göschener Kaltphase I anzusetzen (ZOLLER et al. 1966, 160). ${ }^{\prime}$

\section{0-700 BP}

Die Gliederung in diesem Zeitabschnitt stützt sich vorwiegend auf glazialmorphologische Befunde und Solifluktionsphasen ab. Gletschervorstöße zeichnen sich nach $2170 \mathrm{BP}$, zwischen $1950 \mathrm{BP}$ und $1800 \mathrm{BP}$, nach $1600 \mathrm{BP}$ sowie nach $1200 \mathrm{BP}$ bis $1000 \mathrm{BP}$ und von $900 \mathrm{BP}$ bis $700 \mathrm{BP}$ ab. Ausgeprägte Bodenbildungsphasen sind um 1600 BP und 1000 BP festzustellen. Diese Gliederung deckt sich weitgehend mit dem Wechsel von Solifluktions- und Bodenbildungsphasen. Im allgemeinen muß dieser ganze Zeitraum eher kühl gewesen sein.

Gletschervorstöße:

Nach 2170 BP: Wallis: Findelen (RÖTHLISBERGER 1976, 63), Rossboden (MÜLLER 1975, 27).

Zwischen 1950 BP bis 1800 BP: Wallis: Fee (BIRCHER 1982), Mt. Miné (röthlisberger 1976, 76), Aletsch (H.RÖTHLISBERGER 1963, 308), Fiescher (RÖTHLISBERGER et al. 1980, 46); Gotthardgebiet Maighels (RENNER 1982, 59); Engadin: Cambrena (BEeler 1977, 179).

Nach 1600 BP: Wallis: Brenay (schneEbeli 1976, 45), Corbassière (schneEbeli 1976, 42), Findelen (RöThLISBERGER 1976, 63), Mt. Miné (RÖTHLiSBERGER 1976, 73ff.), Zmutt (RöThlisberger 1976, 89), Aletsch (RÖTHLISBERGER et al. 1980, 47); Engadin: Cambrena (BEELER 1977, 177).

Nach 1200 BP bis 1000 BP: Wallis: Mt. Durand (SCHNEEBELi 1976, 50), Ferpècle (RÖTHLisbeRgeR 1976, 79), Mt. Miné (Röthlisberger 1976, 76) Findelen (RÖTHLISBERGER 1976, 63), Zinal (RÖTHLISBERGER et al. 1980, 47), Aletsch (RöTHLISBERGER et al. 1980, 47); Gotthardgebiet: Stelliboden und Wittenwasseren (RENNER 1982, 43ff.).

Nach 900 BP bis 700 BP: Wallis: Brenay (sCHNEEBELI 1976, 48), Corbassière (schneEbeli 1976, 42), Mt. Miné (Röthlisberger 1976, 78), Mt. Durand (schneEbeli 1976, 50), Findelen (Röthlisberger 1976, 64), Zinal (RöThlisBerger et al. 1980, 48), Moiry (RÖTHLISBERGer et al. 1980, 48), Schwarzberg (BIRCHER 1982), Aletsch (öSCHGER und RÖTHLISBERGER 1961, 191).

Solifluktion: Solifluktionsphasen von 1900 BP? bis $1400 \mathrm{BP}$, von $1000 \mathrm{BP}$ bis $850 \mathrm{BP}$ ? und nach $820 \mathrm{BP}$. Bodenbildungsphasen von $2200 \mathrm{BP}$ bis $1900 \mathrm{BP}$ ? (mit mindestens einem Unterbruch), $1400 \mathrm{BP}$ bis $1000 \mathrm{BP}$ (sehr ausgeprägt) und um 850 BP (GAMPER 1981, 426/427, GAMPER 1982b; RÖTHLISBERGER 1976, 140/141; SCHNEEBELI 1976, 142/143).

Dendroklimatologie: Warmphase: $1670 \mathrm{BP}$ bis $1595 \mathrm{BP}$. Kaltphasen: von $1890 \mathrm{BP}$ bis $1825 \mathrm{BP}$ und $1760 \mathrm{BP}$ bis

\footnotetext{
1 «Angebliche Klimaschwankungen in spätpostglazialen Pollendiagrammen sind ohne stratigraphisch-geomorphologische Indizien mit äusserster Skepsis aufzufassen. In den allermeisten Fällen sind es wohl anthropogen bedingte Vegetationsveränderungen" (BURGA 1979, 272).
} 


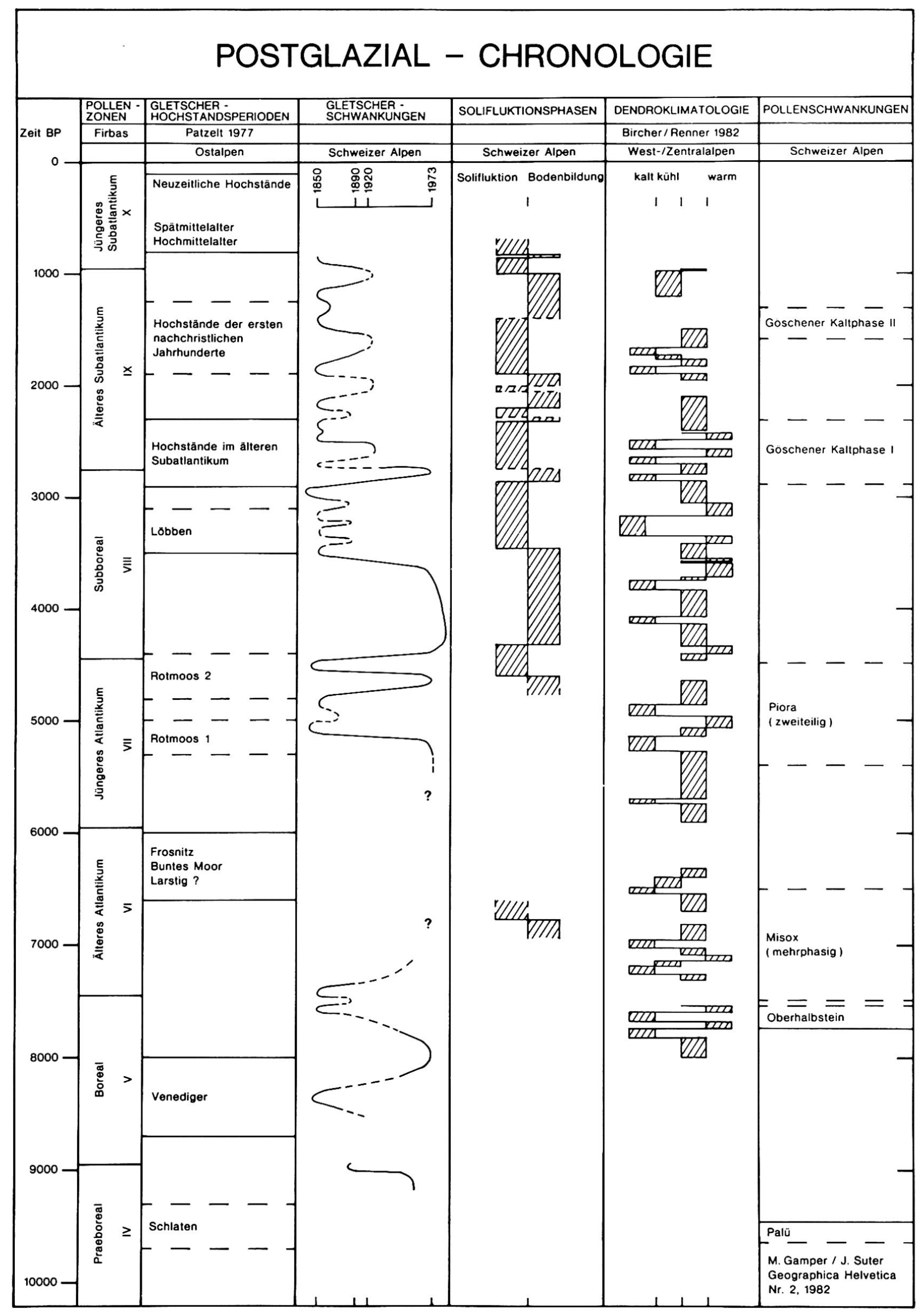


$1670 \mathrm{BP}$; von $1200 \mathrm{BP}$ bis $1030 \mathrm{BP}$ ausgeglichen, aber als Ganzes eher kühl (RENNER 1982, 165).

Pollen: Göschener Kaltphase II 1600 BP bis 1300 BP (zoller et al. 1966, 161). Einzig dieses Ergebnis könnte auf eine Klimaverschlechterung in der
2. Hälfte des älteren Subatlantikums deuten, wobei aber auch hier stratigraphisch-geomorphologische Indizien fehlen.' Anthropogen bedingte Vegetationsveränderungen als Ursache für die Göschener Kaltphasen sind somit nicht völlig auszuschließen.

\section{Zusammenfassung}

In der Figur 1 wird versucht, die zuvor dargelegten Ergebnisse zur postglazialen Klimageschichte der Schweizer Alpen zusammenzufassen. Eine weitergehende Zusammenlegung der vier einzelnen Klimaindikatoren: Gletscherschwankungen, Solifluktionsphasen, Jahrringdichte und Vegetationsänderungen zu einer einzigen Klimakurve scheint uns vorläufig noch nicht möglich. Einmal basiert die wohl detaillierteste Kurve, nämlich die dendroklimatologische Gliederung, auf einer Mischung von Radiocarbondaten und Kalenderjahren. D. h. die einzelnen Jahrringchronologien sind aufgrund der ${ }^{14} \mathrm{C}$-Alter der Hölzer zeitlich eingeordnet, innerhalb der einzelnen Chronologien gelten jedoch Kalenderjahre. Bei längeren Kurven können sich so Verschiebungen gegenüber den anderen Kurven ergeben, welche nur auf konventionellen nicht korrigierten ${ }^{14} \mathrm{C}$-Altern basieren; ein direkter Vergleich der Holzkurve mit den Schwankungen der anderen Klimaindikatoren wird dadurch erschwert. Zudem beinhalten die Holzkurven nur Information über den Temperaturverlauf der Monate Juli, August und September.

Im weiteren sind die Änderungen im Vegetationsbild, wie sie in den pollenanalytischen Untersuchungen erfaßt werden, nicht ohne weiteres mit Gletscherschwankungen zu parallelisieren. Insbesondere in der zweiten Hälfte des Postglazials darf neben den üblichen Interpretationsproblemen der zunehmende menschliche Einfluß auf die Vegetation (z. B. Waldrodungen) nicht unterschätzt werden. Außerdem reagiert die Vegetation träger auf Klimaänderungen als Gletscher, letzteres ist auch bei der Interpretation von Daten zur Höhenlage der Waldgrenze zu berücksichtigen.

Eine weitgehende Vergleichbarkeit besteht einzig zwischen den Gletscherschwankungen und den Solifluktionsphasen. Hier ist jedoch zu beachten, daß die "Informationsdichte» bei der Gletscherkurve bedeutend besser ist als bei den Schwankungen der solifluidalen Aktivität.

Trotz aller dieser Vorbehalte erlauben die vier Kurven in der Abbildung einen generellen Überblick über das Klimageschehen im Postglazial in den Schweizer Alpen: Auffallend ist, da $\beta$ das noch vor wenigen Jahren gültige, einfache Bild des Wechsels von langen kälteren und wärmeren Perioden, wie es sich vor allem aus pollenanalytischen Untersuchungen und aus den meistens auch nur pollenanalytisch belegten Vorstoßperioden der Gletscher der Ostalpen (PATZELT 1977 , in der Abbildung zum Vergleich eingetragen) ergab, einem differenzierteren Klimaverlauf weichen muß mit einem Wechsel von kurzfristigen Pendelungen des Klimas, wie er besonders deutlich in der Holz- und der Gletscherkurve zum Ausdruck kommt. Einzig eine ausgeprägte, längere Warmphase von etwa $4500 \mathrm{BP}$ bis $3600 \mathrm{BP}$ vor heute bildet das Klimaoptimum des Postglazials. Diese Phase der Klimagunst ist durch einen Unterbruch der Solifluktion, das Fehlen von Gletschervorstößen, einen Anstieg der alpinen Waldgrenze über die heutige potentielle Waldgrenze hinaus und mehrere längere Phasen überdurchschnittlicher Dichte in den Holzkurven mehrfach gesichert. $\mathrm{Ab} 3600 \mathrm{BP}$ bis heute scheinen sich in rascher Folge kurze Phasen der Klimaverbesserung und zum Teil recht ausgeprägte, intensive Kaltphasen abgelöst zu haben. Die Extreme dieser Pendelungen können dabei etwa mit den klimatischen Verhältnissen, wie sie in den 40iger und 50iger Jahren unseres Jahrhunderts und während der kleinen Eiszeit (1600-1850) geherrscht haben, verglichen werden. Einzig der Beginn dieser Periode um 3500 BP und der Zeitraum nach 3000 BP dürften noch etwas kälter gewesen sein.

Über den Zeitraum vom Beginn des Postglazials bis um $5400 \mathrm{BP}$ sind wir noch nicht so genau informiert. Die wenigen Holzdichtekurven und Daten zur Gletschergeschichte lassen aber vermuten, da $\beta$ auch in diesem Zeitabschnitt des Postglazials eher mit Verhältnissen wie nach $3600 \mathrm{BP}$ gerechnet werden muß, und $\mathrm{da} B$ das einfachere Bild, wie es sich aus den Pollenschwankungen ergab, auch revidiert werden muß. Dazu sind allerdings noch weitere Untersuchungen notwendig, insbesondere ist $\mathrm{zu}$ hoffen, daß die Holzkurve mit der Zeit noch vervollständigt werden kann. Eine längere Warmphase, wie nach $4500 \mathrm{BP}$, ist in diesem Zeitraum allenfalls von $6300 \mathrm{BP}$ bis $5700 \mathrm{BP}$ wahrscheinlich, aber sicher noch nicht genügend belegt. Zusammenfassend kann festgestellt werden, daß während des ganzen Postglazials im Bereich der Schweizer Alpen sich ein rascher Wechsel von kurzen Phasen der Klimabesserung und zum Teil recht empfindlichen Klimarückschlägen abgespielt hat, nur unterbrochen durch eine längere Warmphase, dem postglazialen Klimaoptimum von $4500 \mathrm{BP}$ bis $3600 \mathrm{BP}$. 


\section{Literaturverzeichnis}

BEELER, F. (1977): Geomorphologische Untersuchungen am Spät- und Postglazial im Schweizerischen Nationalpark und im Berninapaßgebiet (Südrätische Alpen). Ergebnisse der wissenschaftlichen Untersuchungen im Schweizerischen Nationalpark, XV, 131-276.

BEELER, F. (1981): Das Spät- und Postglazial im Berninapaßgebiet. In: Geographica Helvetica, Nr. 3, 101-108.

BEHRE, K. E. (1978): Die Klimaschwankungen im europäischen Präboreal. In: Petermann geogr. Mitt., Nr. 2, 97-102.

BIRCHER, W.: Klima- und Gletschergeschichte des Saastales: Glazialmorphologische und dendroklimatologische Untersuchungen. Physische Geographie, Zürich (im Druck).

BLESS, R. (1982): Postglaziale Schwankungen des Glacier d'Argentière. In: GAMPER, M.: Beiträge zur Quartärforschung in der Schweiz. Physische Geographie, Vol. 1, Zürich.

BORTENSCHLAGER, S. (1977): Ursachen und Ausmaß postglazialer Waldgrenzschwankungen in den Ostalpen. In: FRENZEL, B.: Dendrochronologie und postglaziale Klimaschwankungen in Europa (1977). Erdwissenschaftliche Forschung, XIII, 260-266, Wiesbaden.

BURGA, C. (1979): Postglaziale Klimaschwankungen in Pollendiagrammen der Schweiz. In: Vierteljahresschrift der Naturforschenden Gesellschaft in Zürich, Heft 4: 265-283.

FAEGRI, K. und IVERSEN, J. (1975): Textbook of Pollen Analysis. 3. rev. Aufl. Kopenhagen: Munksgaard, $295 \mathrm{~S}$.

FIRBAS, F. (1949/1952): Spät- und nacheiszeitliche Waldgeschichte Mitteleuropa, Jena.

FRENZEL, B. (1975): The distribution patterns of Holocene climatic changes in the Northern Hemisphere. In: Proc. WMO/IAMAP Sympos. on Long-Term Climat. Fluctuat., WMO N. $421,105-112$.

FRENZEL, B. (1977): Postglaziale Klimaschwankungen im südwestlichen Mitteleuropa. EF 13: 297-322.

FURRER, G. (1954): Solifluktionsformen im Schweizerischen Nationalpark, Ergebn. d. wiss. Unters. im Schweiz. Nationalpark, 4, 203-276.

FURRER, G. (1972): Bewegungsmessungen auf Solifluktionsdecken. In: Z. Geomorph. N. F., Suppl. Bd. 13, 87-101.

FURRER, G. (1977): Klimaschwankungen im Postglazial im Spiegel fossiler Böden: Ein Versuch im Schweizerischen Nationalpark. In: FRENZEL, B.: Dendrochronologie und postglaziale Klimaschwankungen in Europa. Erdwissenschaftliche Forschung 13, 267-270.

FURRER, G., BACHMANN, F. (1972): Solifluktionsdecken im schweizerischen Hochgebirge als Spiegel der postglazialen Landschaftsentwicklung. In: Z. Geomorph., N. F., Suppl. Bd.13, 163-172.

FURRER, G., BACHMANN, F. und FITZE, P. (1971): Erdströme als Formelemente von Solifluktionsdecken im Raum Munt Chavagl/Munt Buffalora (Schweizerischer Nationalpark). Ergebn. d. wiss. Unters. im Schweiz. Nat.-Park. 11, 189-269.

FURRER, G., GAMPER, B. und SUTER, J. (1980): Zur Geschichte unserer Gletscher in der Nacheiszeit - Methoden und Ergebnisse. In: ÖSCHGER, $H$. und MESSERLI, B. und SVILAR, M.: Das Klima - Analysen und Modelle, Geschichte und Zukunft, Berlin.

FURRER, G., LEUZINGER, H. und AMMANN, K. (1975): Klimaschwankungen während des alpinen Postglazials im Spiegel fossiler Böden. In: Vierteljahresschrift d. Naturf. Ges. Zürich, 120, 15-31.
GAMPER-SCHOLLENBERGER, B. (in Vorbereitung): Gletschergeschichte des Oberengadins: Untersuchungen von Gletscherschwankungen in der Bernina- und Languardgruppe.

GAMPER, M. (1976): Bewegungs- und Temperaturmessungen an Erdströmen am Munt Buffalora (Schweizerischer Nationalpark). Diplomarbeit, Manuskript Univ. Zürich.

GAMPER, M. (1981): Heutige Solifluktionsbeträge von Erdströmen und klimamorphologische Interpretation fossiler Böden. Ergebnisse der wissenschaftlichen Untersuchungen im Schweizerischen Nationalpark, XV, 79, 355-443.

GAMPER, M. (1982a): Ein Vergleich der ${ }^{14} \mathrm{C}$-Alter von Huminsäure, organischer Restsubstanz und Holz aus fossilen Böden. In: Bull. der Bodenkundlichen Gesellschaft der Schweiz, 6.

GAMPER, M. (1982b): Postglaziale Solifluktionsphasen am Albulapaß (östliche Schweizer Alpen). In: GAMPER, M.: Beiträge zur Quartärforschung in der Schweiz. Physische Geographie, Vol. 1, Zürich.

GAMPER, M. und OBERHÄNSLI, H. (1982): Interpretation von Radiocarbondaten fossiler Böden. In: GAMPER, M.: Beiträge zur Quartärforschung in der Schweiz. Physische Geographie, Vol. 1, Zürich.

GENSLER, G. A. (1978): Das Klima von Graubünden. Arbeitsber. d. Schweiz. Meteor. Zentralanstalt Zürich, 77.

HAGER, P. K. (1916): Verbreitung der wildwachsenden Holzarten im Vorderrheintal GR. Bern.

HANTKE, R. (1978): Eizeitalter, Band 1, Thun.

HEITZ, C. (1975): Vegetationsentwicklung und Waldgrenzschwankungen des Spät- und Postglazials im Oberhalbstein (Graubünden, Schweiz) mit besonderer Berücksichtigung der Fichteneinwanderung. In: Beitr. zur Geobotan. Landesaufnahme der Schweiz, 55.

HOINKES, H. (1970): Methoden und Möglichkeiten von Massenhaushaltsstudien auf Gletschern. In: Zeitschrift für Gletscherkunde und Glazialgeologie, Heft 1-2: 37-90.

KING, L. (1974): Studien zur postglazialen Gletscher- und Vegetationsgeschichte des Sustenpaßgebietes. Basler Beiträge z. Geogr., H. 18, 125 S., Basel.

KUHN, M. (1979): Climate and glaciers. In: Sea Level, Ice and Climatic Change (Proceedings of the Canberra Symposium, December 1979). IAHS Publ., 131, 3-20.

KUHN, M. (1981): Abschätzung des Energie- und Massenhaushalts von Gletschern in verschiedenen Klimazonen. Vortrag vom 17.12.81 am Geographischen Institut der ETH-Zürich.

KÜTTEL, M. (1977): Pollenanalytische und geochronologische Untersuchungen zur Piottino-Schwankung an der Bedrina (1235 m), Tessin, Schweiz. In: Boreas, 6, 259-274.

KÜTTEL, M. (1979): Kritische Anmerkungen zu Nachweisen präborealer Klimaschwankungen. In: Peterm. Geogr. Mitt. 3, 191-193.

LANDOLT, E. (1969): Unsere Alpenflora. Zürich.

LANG, G. (1952): Zur späteiszeitlichen Vegetations- und Florageschichte Südwestdeutschlands. In: Flora, 139, 243-294.

LANG, G. (1970): Florengeschichte und mediterran-mitteleuropäische Florenbeziehungen. In: Feddes Repert., 81, H.1-5, 315-335.

MAISCH, M. (1981): Glazialmorphologische und Gletschergeschichtliche Untersuchungen im Gebiet zwischen Landwasserund Albulatal (Kt. Graubünden, Schweiz). Physische Geographie, Vol. 3, Zürich.

MÜLLER, F., CAFLISCH, T. und MÜLLER, G. (1976): Firn und Eis der Schweizer Alpen. Geographisches Institut ETH, Publ. Nr. 57, Zürich. 
MÜLLER, H.-N. (1975): Fossile Böden (fAh) in Moränen (Gäli Egga, Rossbodengebiet Simplon VS). In: Bull. de la Murithienne, 92, 21-31.

OESCHGER, $H$. und RÖTHLISBERGER, H. (1981): Datierung eines ehemaligen Standes des Aletschgletschers durch Radioaktivitätsmessungen an Holzproben und Bemerkungen zu Holzfunden an weiteren Gletschern. Zeitschrift für Gletscherkunde und Glazialgeologie, 4/3, 191-205.

PATERSON, W. S. B. (1969): The Physics of Glaciers. Oxford.

PATZELT, G. (1972): Die spätglazialen Stadien und postglazialen Schwankungen von Ostalpengletschern. In: Ber. der Deutschen Botan. Gesellschaft. Bd. 85, Heft 1-4.

PATZELT, G. (1977): Der zeitliche Ablauf und das Ausmaß postglazialer Klimaschwankungen in den Alpen. In: FRENZEL, B.: Dendrochronologie und postglaziale Klimaschwankungen in Europa. Erdwissenschaftliche Forschung 13, 248-259.

PATZELT, G. und BORTENSCHLAGER, S. (1973): Die postglazialen Gletscher- und Klimaschwankungen in der Venedigergruppe (Hohe Tauern, Ostalpen). In: Z. Geomorph., N. F. Suppl. Bd. 16, 25-72.

RENNER, F. (1982): Beiträge zur Gletschergeschichte des Gotthardgebiets und dendroklimatologische Analysen an fossilen Hölzern. Physische Geographie, Vol. 8, Zürich.

RÖTHLISBERGER, F. (1976): Gletscher- und Klimaschwankungen im Raum Zermatt, Ferpècle und Arolla. In: Die Alpen 52, 59-152.

RÖTHLISBERGER, F. et al. (1980): Holocene Climatic Fluctuations - Radiocarbon Dating of fossil Soils (fAh) and Woods from Moraines and Glaciers in the Alps. In: MÜLLER, F., BRIDEL, L. und SCHWABE, E.: Geography in Switzerland. Geographica Helvetica, 5, 21-52.

HARRER Heinrich: Der Himalaya blüht. 153 S., Fotos, Umschau-Verlag Frankfurt/M, 1980, DM 58.--

DARGYAY Eva: Ladakh. Innenansicht eines Landes. 300 S., Fotos, Diederichs Düsseldorf, 1980, DM 36.-..

AHLUWALIA H.P.S.: Hermit kingdom Ladakh. 186 S. Fotos, Karten, Vikas Publishing House New Delhi, 1980, 295 Rupees.

Die Lücke, welche der Rückzug der britischen Autoren aus Indien geschaffen hatte, wird geschlossen. Gute drei Jahrzehnte war der Himalaya-Reisende auf längst vergriffene Literatur angewiesen, die sich bei ausgiebigem Suchen in der herrlichen Unübersichtlichkeit indischer Buchhandlungen manchmal zufällig noch finden liess. Dann folgte die Epoche der Reprints, welche wenigstens einige Klassiker wieder allgemein zugänglich machte. Inzwischen aber sind neue Werke aufgetaucht, sowoh1 von europäischen wie landeseigenen Autoren.

HARRER bietet dem etwas, der von allem ein biss-
RÖTHLISBERGER, H. (1963): In: Radiocarbon, 5, 308.

SCHNEEBLI, W. (1976): Untersuchungen von Gletscherschwankungen im Val de Bagnes. In: Die Alpen 52, 5-57.

SCHWEINGRUBER, F. (1976): Röntgenuntersuchungen an Jahrringen. In: Neue Zürcher Zeitung, 180, 34.

SCHWEINGRUBER, F. et al. (1980): The X-Ray Technique as applied to Dendroclimatology. In: Tree-Ring Bulletin, 38, 61-91.

SCHWEINGRUBER, F. et al. (1979): Dendroclimatic studies on conifers from central Europe and Great Britain. In: Boreas, 8 , 427-452.

SUTER, J. (1981): Gletschergeschichte des Oberengadins: Untersuchungen von Gletscherschwankungen in der Err-JulierGruppe. Physische Geographie, Vol. 2, Zürich.

SUTER, J. (1982): Zur klimatischen Interpretation der Gletschergeschichte. In: GAMPER, M.: Beiträge zur Quartärforschung in der Schweiz. Physische Geographie, Vol. 1, Zürich.

ZOLLER, H. (1960): Pollenanalytische Untersuchungen zur Vegetationsgeschichte der insubrischen Schweiz. Denkschrift der Schweiz. Naturf. Ges., Band LXXXIII, Abh. 2, 112 S.

ZOLLER, H. (1968): Postglaziale Klimaschwankungen und inr Einfluß auf die Waldentwicklung Mitteleuropas einschließlich der Alpen. In: BDBG, 80, H. 10: 690-691.

ZOLLER, H. (1977): Alter und Ausmaß postglazialer Klimaschwankungen in den Schweizer Alpen. In: FRENZEL, B.: Dendrochronologie und postglaziale Klimaschwankungen in Europa. In: Erdwissenschaftliche Forschung 13, 271-281.

ZOLLER, H., SCHINDLER, C. und RÖTHLISBERGER, H. (1966): Postglaziale Gletscherstände und Klimaschwankungen im Gotthardmassiv und Vorderrheingebiet. In: Verh. Natf. Ges. Basel, 77, H. 2, 97-164. chen wissen möchte, etwas Botanik, etwas Volkskunde, etwas Reisebeschreibung. Der Fachmann braucht natürlich mehr. Wer aber über sein Interessengebiet hinaus in anderen Disziplinen schnuppern möchte, für den ist HARRER eben recht.

Frau DARGYAY kennt als Tibetologin den Ladakh und seine Leute gründlich. Wer ihr Buch vor dem Betreten des Landes liest, ist von der fülle erschlagen. Hinterher aber ist die Lektüre $\mathrm{Ge}-$ winn. Fast alle offenen Fragen finden sich beantwortet, aus welchem Gebiet auch immer. Das Ladakh-Buch des Majors AHLUWALIA crreicht drucktechnisch vielleicht noch nicht restlos den Stand europäischer Imprimate, die Fortschritte sind aber unübersehbar. Dieses Werk orientiert über wenig zugängliche Gebiete und stellt jüngere Entwicklungen aus den Augen eines Landmanns dar; für den Reisenden aus dem Westen ein nützliches Unterfangen! Es ist wie die meisten indischen Bücher über D.K. Agencies, 313/74-D, Inderlok, Old Rohtak Rd, Delhi 110035, erhältlich.

GH 2/82 Walter Kyburz, Zürich 